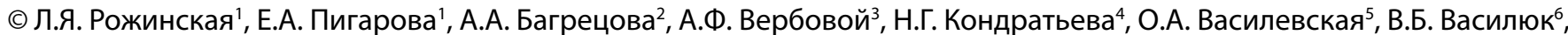
М.В. Манько ${ }^{7}$, В.Б. Шуньков ${ }^{8}$, Т.А. Гребенникова ${ }^{1}$

'ФГБУ «НМИЦ эндокринологии» Минздрава России, Москва

${ }^{2}$ ФГБУЗ «Северный медицинский клинический центр им Н.А. Семашко ФМБА», Архангельск

${ }^{3} \mathrm{OOO}$ «Центр «Диабет»», Самара

${ }^{4} \mathrm{OOO}$ «Аврора», Санкт-Петербург

${ }^{5} \mathrm{OOO}$ «Медицинский диагностический центр», Ярославль

${ }^{6} \mathrm{OOO}$ «Научно-исследовательский центр Экобезопасность», Санкт-Петербург

73АО «Наследники», Москва

вчУЗ «Клиническая больница «РЖД-Медицина», Санкт-Петербург

Обоснование. Низкий уровень витамина D имеет серьезные медицинские, социальные и экономические последствия для здоровья человека и требует проведения своевременной диагностики и адекватной коррекции.

Цель. Оценка эффективности и безопасности терапии препаратом Фортедетрим, капсулы мягкие («МЕДАНА ФАРМА» AO, Польша) при использовании насыщающих и поддерживающих доз в сравнении с терапией препаратом Вигантол ${ }^{\circledR}$, раствор для приема внутрь масляный (Мерк КГаА, Германия) у пациентов с дефицитом витамина D.

Материалы и методы. В исследование были включены 150 пациентов с дефицитом витамина D (менее 20 нг/мл) в возрасте от 18 до 75 лет, которые были рандомизированы в 3 группы в соотношении 1:1:1 (по 50 человек). В двух группах в течение первых 4 нед использовали капсулы Фортедетрим соответственно $50000 \mathrm{ME} 1$ раз в неделю (5 капсул по 10000 ME) и 56000 ME — по 8000 ME ежедневно (2 капсулы по 4000 ME), а в группе сравнения — Вигантол ${ }^{\circledR}$ по 1000 ME ежедневно (2 капли); в течение последующих 12 нед пациенты получали поддерживающую терапию в первой группе применяли Фортедетрим 10000 ME 1 раз в неделю, во второй - Фортедетрим по 2000 МЕ ежедневно (14 000 МЕ в неделю); в группе сравнения продолжали прием Вигантола ${ }^{\circledR} 1000$ МЕ ежедневно.

Результаты. В результате оценки данных по результатам I и II этапов проведенного клинического исследования Фортедетрима в сравнении с Вигантолом ${ }^{\circledast}$ была доказана гипотеза о превосходстве высокодозного препарата Фортедетрим (капсулы по 2000, 4000 и 10000 ME) над препаратом Вигантол ${ }^{\circledR}$ (капли, 1000 МЕ ежедневно), с эквивалентной безопасностью лечения. Тем не менее необходимо отметить, что и длительный прием колекальциферола в дозе 1000 ME ежедневно к 20 нед позволил достичь уровня 30 нг/мл у 36\% участников, но это значимо меньше, чем в группах с применением высокодозных препаратов.

Различий в количестве нежелательных явлений за время исследования, их степени, связи с приемом препаратов между группами лечения и группой сравнения не выявлено.

Заключение. Использование препарата Фортедетрим в насыщающих дозах является эффективным и безопасным для лечения взрослых пациентов с дефицитом витамина D. Тем не менее необходим индивидуальный подход к его восполнению (с учетом комплаентности, степени ожирения, нарушений всасываемости кальция и витамина D), так как не все пациенты в примененных режимах насыщения достигли целевых значений витамина D в крови.

КЛЮЧЕВЫЕ СЛОВА: колекальциферол; витамин D; кальций

\title{
HIGH-DOSES OF CHOLECALCIFEROL FOR VITAMIN D DEFICIENCY TREATMENT: RESULTS OF AN OPEN-LABEL, MULTICENTER, COMPARATIVE, RANDOMIZED TRIAL
}

(c) Liudmila Y. Rozhinskaya', Ekaterina A. Pigarova', Anastasia A. Bagretsova², Andrey F. Verbovoy ${ }^{3}$, Natalia G. Kondratyeva ${ }^{4}$, Olga A. Vasilevskaya ${ }^{5}$, Vasily B. Vasilyuk ${ }^{6}$, Maria V. Manko ${ }^{7}$, Victor B. Shunkov ${ }^{8}$, Tatiana A. Grebennikova ${ }^{1}$

'The National Medical Research Center for Endocrinology, Moscow, Russia

${ }^{2}$ Northern Medical Clinical Center named after N.A. Semashko, Arkhangelsk, Russia

${ }^{3}$ «Diabetes Center» LLC, Samara, Russia

${ }^{4}$ «Aurora» LLC, St. Petersburg, Russia

${ }^{5}$ LLC «The Medical diagnostic center», Yaroslavl, Russia

${ }^{6} \mathrm{LLC}$ «Research Center Eco-safety», St. Petersburg, Russia

${ }^{7}$ CJSC «Heirs», Moscow, Russia

${ }^{8}$ Private educational institution «Clinical hospital» Russian Railways-Medicine «of St. Petersburg», St. Petersburg, Russia 
Background: Vitamin D deficiency have serious medical, social and economic consequences for human health and require timely diagnosis and adequate correction.

Aim: Evaluation of the efficacy and safety of therapy with Fortedetrim soft capsules (MEDANA PHARMA AO, Poland) using saturating and maintenance doses compared to therapy with Vigantol ${ }^{\circledR}$, an oily solution for oral administration (Merck KGaA, Germany) in patients with vitamin D deficiency.

Materials and methods: The study included 150 patients with vitamin D deficiency (less than $20 \mathrm{ng} / \mathrm{ml}$ ) aged 18 to 75 years, who were randomized into 3 groups in a 1:1:1 ratio (50 people each). Fortedetrim capsules were used in two groups during the first four weeks 50,000 IU once a week (5 capsules 10,000 IU each) and 56,000 IU - 8,000 IU daily (2 capsules $4000 \mathrm{IU}$ ), and Vigantol ${ }^{\circledR}$ was used in the comparison group $1000 \mathrm{IU}$ daily ( 2 drops). Over the next 12 weeks, patients received supportive therapy - in the first group, Fortedetrim 10,000 IU was used once a week, in the second - Fortedetrim 2,000 IU daily $\left(14,000\right.$ IU per week); the comparison group continued to take Vigantol ${ }^{\circledR} 1000$ IU daily.

Results: As a result of evaluating the data on the results of stages I and II of the clinical study Fortedetrim in comparison with Vigantol $^{\circledR}$, the hypothesis was proved that the high-dose drug Fortedetrim (capsules of 2000, 4000 and $10000 \mathrm{IU}$ ) is superior to the drug Vigantol ${ }^{\circledast}$ (drops, $1000 \mathrm{IU}$ daily). Nevertheless, it should be noted that long-term intake of colecalciferol at a dose of 1000 IU daily by 20 weeks allowed $36 \%$ of participants to reach a level of $30 \mathrm{ng} / \mathrm{ml}$, but this is significantly less than in groups using high-dose drugs. There were no differences in the number of adverse events during the study, their degree and the relationship with drug intake between the treatment and comparison groups.

Conclusion: The use of the drug Fortedetrim in saturating doses is effective and safe for the treatment of adult patients with vitamin D deficiency. However, an individual approach to its replenishment is required (taking into account compliance, the degree of obesity, impaired absorption of calcium and vitamin D), since not all patients in the applied saturation regimes, the target values of vitamin $D$ in the blood were reached.

KEYWORDS: cholecalciferol; vitamin D; calcium

\section{ОБОСНОВАНИЕ}

Bo многих странах мира пандемия дефицита витамина D является глобальной проблемой здравоохранения [1]. Низкий статус витамина D имеет серьезные медицинские, социальные и экономические последствия для здоровья человека и требует проведения своевременной диагностики и адекватной коррекции [2]. Для оценки уровня витамина D используется уровень 25OHD (кальцидиола) в сыворотке крови, который отражает суммарное количество витамина $D$, поступившего в организм человека как с пищевыми продуктами, так и синтезированного в коже под воздействием солнечной инсоляции [3]. Кальцидиол является функциональным индикатором для определения уровня витамина D. B связи с этим оправдан неподдельный интерес исследователей к изучению различных схем коррекции дефицита и недостаточности витамина D как для эффективного лечения, так и для удобства пациента и их долгосрочной приверженности к терапии. В настоящее время в Российской Федерации принято несколько подходов к терапии дефицита и недостаточности витамина D [4], однако до сих пор не определен приоритетный и оптимальный алгоритм лечения. Анализируя данные исследований последних лет, отмечена тенденция по изучению высоких доз витамина D и их влияния не только на костную ткань [5], но и на другие органы и системы $[6,7,8]$ организма человека. Дозы, использованные в этих исследованиях, варьировались от 50000 до $400000 \mathrm{ME}$, а период наблюдения от 28 дней, до нескольких лет. Так например, Schleck M.L. et al. провели по оценке эффективности терапии высокими дозами витамина D, в котором колекальциферол назначался сначала в дозе насыщения в течение 3 мес (50 000, 100000 и 200000 ME 1 раз в месяц) с переводом на поддерживающую дозу (25 000, 50000 и 100000 МЕ соответственно 1 раз в месяц), что в течение следующих 2 мес (общая доза составила 100 000, 200000 и 400000 MЕ) привело к достоверному и быстрому увели- чению уровня 25OHD [9]. Авторы наблюдали дозозависимое повышение уровня 25OHD в сыворотке крови. При этом они не отметили нежелательных явлений за весь период исследования, а также не зафиксировали случаев токсичности витамина D. B работе Ilahi M. оценена эффективность и безопасность влияния большой пероральной дозы колекальциферола. Согласно результатам, сывороточный кальцидиол быстро повышался после введения колекальциферола. Для контроля безопасности у пациентов производился забор общего кальция исходно и на 1, 3, 5 и 112-е сутки, а также уровень паратиреоидного гормона (ПТГ) был определен в начале и в конце исследования. При этом сывороточный кальцидиол быстро повышался после введения колекальциферола от среднего исходного уровня до концентрации максимума и не превышал уровня, принятого за нормативный интервал [10]. Анализируя вопрос безопасности назначения витамина D Dudenkov D.V. и соавт. отметили, что, несмотря на то, что за время лечения возросло количество лиц с уровнем 25(OH)D выше 50 нг/мл почти в 20 раз, был обнаружен только один случай, сопровождающийся гиперкальциемией, когда уровень витамина D был выше 364 нг/мл [11]. Cannell J.J. и Hollis B.W. в своей статье утверждают, что для возникновения гиперкальциемии необходимо принимать витамин D в дозе, превышающей 10000 ME в сутки, в течение многих месяцев или даже лет [12]. Таким образом, отсутствие единого подхода в лечении недостаточности и дефицита витамина D явилось стимулом для детального изучения вопросов насыщения и регуляции поддерживающих доз при разных режимах его введения. Также необходимо отметить, что к началу проведения исследования в России в качестве медицинских препаратов были доступны только 2 формы колекальциферола: Аквадетрим и Вигантол ${ }^{\circledR}$ в каплях по 500 ME в 1 капле, что мало и неудобно для применения препаратов в насыщающих дозах и для лечения в поддерживающих дозах ряда пациентов, нуждающихся в повышенных дозировках 
препарата (например при ожирении, остеопорозе, приеме глюкокортикоидов и т.д.), что и послужило актуальностью для проведения данного исследования.

\section{ЦЕЛЬ ИССЛЕДОВАНИЯ}

Оценка эффективности и безопасности терапии препаратом Фортедетрим, капсулы мягкие («МЕДАНА ФАРМА» AO, Польша) при использовании насыщающих и поддерживающих доз в сравнении с терапией препаратом Вигантол ${ }^{\circledast}$, раствор для приема внутрь масляный («Мерк КГаА», Германия) у пациентов с дефицитом витамина D.

\section{МАТЕРИАЛЫ И МЕТОДЫ}

Дизайн исследования и объекты. Открытое многоцентровое сравнительное рандомизированное исследование III фазы. Центры, принявшие участие в данном исследовании, приведены в таблице 1 .

В табл. 2 представлены дизайн исследования и его этапы. Исследование проводилось в период с 01.03.2018 по 30.05.2019.

Пациенты, соответствующие всем критериям включения и не соответствующие ни одному из критериев невключения, были рандомизированы в 3 группы в соотношении 1:1:1 (по 50 человек)

Исследование проводилось в соответствии с требованиями GCP. Копия протокола предоставлялась на рас- смотрение в Независимый междисциплинарный Комитет по этической экспертизе клинических исследований. Исследование было начато после получения одобрения этического комитета и выполнения необходимых процедур в соответствии с требованиями официальных инстанций.

Критерии включения в исследование.

1. Пациенты мужского и женского пола в возрасте от 18 до 75 лет включительно на момент подписания Формы информированного согласия ИЛП.

2. Наличие подписанной Формы информированного согласия ИЛП на участие в клиническом исследовании.

3. Дефицит витамина D: концентрация 25OHD в сыворотке крови $<20$ нг/мл на момент скрининга.

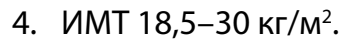

5. Согласие пациента на использование надежных методов контрацепции на протяжении всего исследования и в течение 30 дней после его завершения. В исследовании могли принять участие: женщины, имеющие отрицательный тест на беременность и использующие следующие средства контрацепции: барьерный метод или двойной барьерный метод контрацепции. В исследовании также могли принять участие женщины, неспособные к деторождению (в анамнезе: гистерэктомия, перевязка маточных труб, бесплодие, менопауза более 1 года). или мужчины с сохранной репродуктивной функцией, использующие барьерные средства контрацепции, а также мужчины с бесплодием или перенесенной вазэктомией в анамнезе.

Таблица 1. Центры, принявшие участие в исследовании

\section{Центры - участники исследования}

ФГБУ «НМИЦ эндокринологии» Минздрава России (гл. исследователь - Л.Я. Рожинская)

ФГБУЗ «Северный медицинский клинический центр им Н.А. Семашко» ФМБА (гл. исследователь - А.А. Багрецова)

ООО «Центр «Диабет»» (гл. исследователь - А.Ф. Вербовой)

ООО «Аврора» (гл. исследователь - Н.Г. Кондратьева)

ООО «Медицинский диагностический центр» (гл. исследователь - О.А. Василевская)

ООО «Научно-исследовательский центр Экобезопасность» (гл. исследователь - В.Б. Василюк)

ЗАО «Наследники» (гл. исследователь - М.В. Манько)

НУЗ «Дорожная клиническая больница открытого акционерного общества «РЖД» (гл. исследователь - В.Б. Шуньков)

Табл. 2. Дизайн исследования и его этапы

\begin{tabular}{|c|c|c|c|}
\hline Группы & $\begin{array}{l}\text { I этап } \\
\text { (8 нед) }\end{array}$ & $\begin{array}{l}\text { Достигнута } \\
\text { нормализация } \\
250 H D ? \\
\text { Да/нет }\end{array}$ & 2 этап, 12 нед \\
\hline \multirow[b]{2}{*}{ 1. Фортедетрим } & \multirow{2}{*}{$\begin{array}{l}50000 \mathrm{ME} \\
1 \text { раз } \\
\text { в неделю } \\
\text { (5 капсул } \\
\text { по } 10000 \mathrm{ME})\end{array}$} & ДА & Поддерживающая доза 10000 ME (1 капсула 1 раз в неделю) \\
\hline & & HET & $\begin{array}{l}50000 \text { ME } 1 \text { раз в неделю (5 капсул по } 10000 \text { ME) до } \\
\text { нормализации уровня витамина D (минимум } 4 \text { нед), далее } \\
\text { поддерживающая доза } 10000 \text { ME (1 капсула } 1 \text { раз в неделю) } \\
\text { до } 12 \text { нед включительно }\end{array}$ \\
\hline \multirow[b]{2}{*}{ 2. Фортедетрим } & \multirow{2}{*}{$\begin{array}{l}8000 \text { ME } \\
\text { ежедневно } \\
\text { ( } 2 \text { капсулы } \\
\text { по } 4000 \text { ME) }\end{array}$} & ДА & $\begin{array}{l}\text { Поддерживающая доза } 14000 \text { ME (1 капсула по } 2000 \text { ME } 1 \text { раз } \\
\text { в день ежедневно) }\end{array}$ \\
\hline & & HET & $\begin{array}{l}8000 \text { ME ежедневно (2 капсулы по } 4000 \text { ME) до нормализации } \\
\text { уровня витамина D (минимум } 4 \text { нед), далее поддерживающая } \\
\text { доза по } 14000 \text { ME (1 капсула по } 2000 \text { ME } 1 \text { раз в день ежедневно) }\end{array}$ \\
\hline \multirow{2}{*}{ 3. Вигантол ${ }^{\circledR}$} & \multirow{2}{*}{$\begin{array}{l}1000 \mathrm{ME} \\
\text { ежедневно } \\
\text { (по } 2 \text { капли) }\end{array}$} & ДА & 2 капли (1000 ME ежедневно) до 12 нед включительно \\
\hline & & HET & 2 капли (1000 ME ежедневно) до 12 нед включительно \\
\hline
\end{tabular}




\section{Критерии невключения.}

В исследование не включались пациенты, имеющие хотя бы один из следующих критериев.

1. Повышенная чувствительность к компонентам исследуемых препаратов/препарата сравнения.

2. Гиперкальциемия на момент скрининга.

3. Длительная (более 2 мес) иммобилизация в период участия пациента в исследовании.

4. Тяжелые формы сердечно-сосудистых заболеваний (III-IV степень по классификации Нью-Йоркской Ассоциации Кардиологов (NYHA)), в том числе нестабильная прогрессирующая стенокардия IV ФК, неконтролируемая артериальная гипертензия.

5. Хроническая почечная недостаточность (скорость клубочковой фильтрации <60 мл/мин) на момент скрининга.

6. Заболевания, сопровождающиеся гиперкальциурией (например, Болезнь Педжета, нефрокальциноз, нефроуролитиаз, псевдогипопаратиреоз, саркоидоз), на момент скрининга.

7. Клинические признаки синдрома мальабсорбции на момент скрининга (например, диарея, боли в животе, астеновегетативный синдром и т.д.).

8. Глютеновая болезнь в анамнезе.

9. Воспалительные заболевания кишечника (язвенный колит, болезнь Крона).

10. Муковисцидоз.

11. Прием любых препаратов или пищевых добавок, содержащих витамин D, в течение 2 мес до скрининга.

12. Применение гормонозаместительной терапии (ГЗТ) женскими половыми гормонами в течение 3 мес до скрининга.

13. Прием системных глюкокортикостероидов (ГКС) в течение 3 мес до скрининга (допускался однократный прием).

14. Прием в анамнезе более чем 10 ед. алкоголя в неделю (1 ед. алкоголя эквивалентна 500 мл пива, 200 мл вина или 50 мл крепких спиртных напитков) или анамнестические сведения/данные об алкоголизме, наркомании, злоупотреблении лекарственными препаратами.

15. Ожидаемое или планируемое увеличение экспозиции солнечными лучами (например, планируемая поездка в «солнечные страны» или на море, пляжный отдых) или нежелание отказаться от длительного нахождения на солнце в период участия в исследовании.

16. Сдача или потеря крови объемом более 300 мл (включая плазмаферез) или переливание любых компонентов крови в течение 3 мес до скрининга.

17. Планируемое в ближайшие 6 мес или перенесенное за последние 3 мес до скрининга обширное хирургическое вмешательство (связанное с риском для жизни пациента).

18. Синдром печеночной недостаточности, подтвержденный данными объективного осмотра и данными клинико-лабораторных исследований (активность аланинаминотрансферазы и/или аспартатаминотрансферазы 21,5×верхнюю границу норму (ВГН), концентрация общего билирубина $\geq 1,5 \times B$ Г) на момент скрининга.

19. Тяжелые, декомпенсированные или нестабильные соматические заболевания (любые заболевания или состояния, которые угрожают жизни пациента или ухудшают прогноз пациента, а также делают невозможным его участие в клиническом исследовании).
20. Острые воспалительные заболевания или хронические воспалительные заболевания в стадии обострения на момент скрининга.

21. Беременность или период грудного вскармливания.

22. Неспособность пациента, по мнению врача-исследователя, выполнить условия данного протокола.

23. Участие в другом клиническом исследовании в течение 3 мес до скрининга.

24. Прочие условия, которые, по мнению врача-исследователя, препятствуют включению пациента в исследование.

\section{Запрещенная терапия/процедуры.}

- Рифампицин.

- Изониазид.

- Витамин А.

- Барбитураты (например, фенобарбитал).

- Тиазидные диуретики (например, гидрохлоротиазид и др.).

- Системные ГКС (например, беклометазон, дексаметазон, будесонид, преднизолон).

- Противосудорожные препараты (например, фенитоин, примидон).

- Антипсихотические препараты (например, хлорпромазин, галоперидол, арипипразол, кветиапин, рисперидон).

- Препараты, изменяющие (снижающие) абсорбцию жира в желудочно-кишечном тракте (например, колестипол, орлистат, колестирамин).

- Осмотические (например, лактулоза, макрогол) и солевые (например, натрия пикосульфат, магния сульфат) слабительные средства, а также касторовое, оливковое, миндальное, вазелиновое и другие масла, применяемые с целью достижения слабительного эффекта.

- Препараты, которые ингибируют (например, кетоконазол, амиодарон) или индуцируют (например, карбамазепин, омепразол) активность ферментов системы СYР450 (в течение I этапа исследования).

- Гиполипидемическое средство - ингибитор абсорбции холестерина (эзетимиб).

- Посещение солярия.

- Применение пищевых добавок или препаратов кальция, содержащих >500 мг кальция/день.

- Любые препараты или пищевые добавки, содержащие витамин D, кроме исследуемых препаратов/препарата сравнения.

- Бисфосфонаты.

- Препараты, влияющие на концентрацию ПтГ (терипаратид, цинакальцет).

Критерии оценки эффективности.

Оценка эффективности терапии осуществлялась по следующим точкам.

Первичная конечная точка эффективности. Величина изменения концентрации 25OHD в крови пациентов к Визиту 3 относительно Визита 1.

Вторичные конечные точки эффективности.

Динамика концентрации 25OHD с 1 по 3 визиты (Этап I).

Динамика концентрации 25OHD с 4 по 6 визиты (Этап II).

Количество пациентов (в \%), у которых отмечается повышение концентрации 250HD $\geq 20$ нг/мл в крови к Визитам 2, 3. 
Количество пациентов (в \%), у которых отмечается повышение концентрации 25OHD $\geq 30$ нг/мл к Визитам 2, 3, $4,5,6$.

\section{Конечные точки безопасности:}

общее количество нежелательных явлений (НЯ), стратифицированных по тяжести и частоте;

частота НЯ, связанных с применением исследуемых препаратов/препарата сравнения;

частота серьезных НЯ (СНЯ), связанных с применением исследуемых препарата сравнения;

доля пациентов, у которых зарегистрировано как минимум одно НЯ;

доля пациентов, прервавших лечение из-за возникновения НЯ.

Нежелательным явлением (НЯ) считалось любое неблагоприятное изменение в состоянии здоровья пациента или субъекта клинического исследования, у которого применялся лекарственный препарат, независимо от причинно-следственной связи с его применением

Таким образом, нежелательное явление (НЯ) может представлять собой любой неблагоприятный симптом (включая отклонение лабораторного показателя от нормы), жалобу или заболевание, время возникновения которого не исключает причинно-следственной связи с применением лекарственного (исследуемого) продукта вне зависимости от наличия или отсутствия такой связи.

Степень выраженности НЯ определялась следующим образом:

Легкая: не препятствует стандартной активности.

Умеренная: препятствует стандартной активности.

Тяжелая: пациент не способен к стандартной активности.

Тяжелое нежелательное явление необязательно было «серьезным» по природе, «серьезное» нежелательнее явление не обязательно было «тяжелым» по определению.

Угроза жизни и здоровью.

Смерть.

Методы исследования.

Сбор анамнеза, информации о перенесенных и сопутствующих заболеваниях, о предшествующей терапии, измерение роста и массы тела, регистрация сопутствующей терапии.

Оценка основных жизненно важных показателей (артериальное давление, частота сердечных сокращений, $\left.\mathrm{t}^{\circ} \mathrm{C}\right)$. Физикальное обследование.

ЭКГ (на Визите О ЭКГ проводилась для пачиентов старше 40 лет или у пациентов с установленной ранее патологией сердечно-сосудистой системы).

Клинический анализ крови (гемоглобин, гематокрит, эритроциты, лейкоциты, лейкоцитарная формула (нейтрофилы, эозинофилы, моноциты, базофилы, лимфоциты), тромбоциты, (ОЭ).

Биохимический анализ крови (АСТ, АЛТ, общий белок, креатинин, мочевина, общий билирубин, глюкоза, кальций общий. Определение скорости клубочковой фильтрации (СКФ), ПТГ. На Визитах 0, 3 и 6 дополнительно определялись фосфаты). Все вышеперечисленные анализы и анализы мочи проводились в лабораториях Центров, участвующих в исследовании.

Определение $25 \mathrm{OHD}$ осуществлялось в центральной лаборатории ФБУН «ЦНИИ эпидемиологии» Роспотребнадзора методом иммунохемилюминесцентного анализа на аппарате Diasorin.
После получения результатов анализа крови на 25OHD осуществлялся телефонный звонок пациенту с целью коррекции режима терапии. Для пациентов группы 2, не достигших нормализации концентрации витамина D на Этапе I и продолжающих прием высоких доз исследуемого препарата, выдавался препарат в двух дозировках. После получения результатов анализа крови на 25OHD врач-исследователь информировал пациента о необходимости изменения/сохранения прежнего режима терапии.

Общий анализ мочи (pH, относительная плотность/ удельный вес, белок, глюкоза, эритроциты, лейкоциты; на Визитах 0, 3 и 6 дополнительно проводилось определение кальция). Тест на беременность проводился для женщин, способных к деторождению.

\section{Расчет размера выборки.}

Основная гипотеза исследования состояла в доказательстве превосходства эффективности терапии препаратом Фортедетрим (в двух режимах применения) над терапией препаратом Вигантол ${ }^{\circledR}$. В соответствии в Российскими клиническими рекомендациями по диагностике, профилактике и лечению дефицита витамина D достаточным считается уровень 25OHD более 30 нг/мл [4].

Расчет выборки в PASS1 1 при выборе границы превосходства в 3,197 нг/мл, ожидаемой разницы в 6,72 нг/мл, величины $\mathrm{SD}=5,9$ нг/мл, одностороннего уровня значимости 0,025 и мощности 80\% показал необходимость включения не менее 46 пациентов в каждую группу. Всего с учетом выбывания в настоящее исследование было включено 150 пациентов с дефицитом витамина D, по 50 в каждую из 3 групп.

\section{Статистические методы.}

Демографические данные (возраст, пол), данные исходного состояния были представлены в зависимости от типа переменной при помощи среднего значения, стандартного отклонения, медианы, межквартильного размаха, минимума и максимума или в виде частот и процентного отношения.

Для проверки гипотезы об однородности групп исследования в исходном периоде было проведено тестирование отсутствия различий между группами при помощи t-критерия Стьюдента (для интервальных показателей с нормальным распределением в исследуемой популяции), критерия Манна-Уитни (для ординальных показателей или для интервальных показателей с распределением, отличающимся от нормального) или критерия $X^{2}$ (для качественных признаков). В случае нахождения статистически значимых различий между группами была произведена оценка величины различий между группами исследования при помощи доверительных интервалов.

Анализ эффективности был произведен для популяции пациентов, завершивших I и II этапы исследования. Первичный анализ эффективности был основан на выборках пациентов, завершивших I этап исследования в соответствии с протоколом (РP). Анализ эффективности терапии на II этапе исследования был проведен на основании данных всех включенных в исследование пациентов (ITT). После завершения II этапа дополнительно был проведен совокупный анализ данных всех включенных в исследование пациентов (по популяциям ITT и РP). Для тестирования нулевой гипотезы об отсутствии различий между группами по основному 
показателю эффективности использовался t-критерий Стьюдента для независимых выборок. В том случае, если необходимые исходные допущения о характере распределения данного показателя не были выполнены, использовался непараметрический критерий Манна-Уитни. Все показатели эффективности сравнивались между группами исследования. Для оценки превосходства эффективности исследуемого препарата был использован подход, основанный на построении 95\% доверительного интервала (ДИ) для разницы между величиной изменения основного критерия эффективности в группах (т.е. исследуемый препарат Аквадетрим Форте в дозовом режиме 50000 ME 1 раз в неделю минус препарат Вигантол ${ }^{\circledR}$ по 2 капли 1000 ME в день и исследуемый препарат Аквадетрим Форте в дозовом режиме $8000 \mathrm{ME}$ ежедневно минус препарат Вигантол ${ }^{\circledR}$ по 2 капли 1000 МЕ в день).

Доказательством гипотезы превосходства являлось установление между группами статистически значимой разницы по величине прироста концентрации 25OHD в крови пациентов к Визиту 3. То есть величины $\mathrm{SM} \geq 3,197$ нг/мл, соответствующей клинически значимой разнице между группами пациентов с дефицитом витамина D. Сравнение групп по критериям оценки эффективности по окончании терапии проводилось для непрерывных показателей - с помощью дисперсионного анализа ANOVA (при условии нормального распределения и равенстве дисперсий) или соответствующего непараметрического аналога (ранговый критерий Крускала-Уоллиса для оценки разностей между медианами с поправкой Бонферрони для множественных сравнений). При установлении значимых различий проводилось попарное сравнение групп с применением t-критерия Стьюдента или критерия Манна-Уитни (в зависимости от принятого заключения о характере распределения признака). Оценка частотных показателей эффективности с помощью критерия $\mathrm{X}^{2}$ (для частотных показателей).

Анализ безопасности был проведен на популяции безопасности. Критериями безопасности являлись: общее количество НЯ, стратифицированных по тяжести и частоте, частота нежелательных реакций, частота СНЯ, связанных с применением исследуемого препарата/ препарата сравнения, доля пациентов, у которых зарегистрировано как минимум одно НЯ, доля пациентов, прервавших лечение из-за возникновения НЯ. Было рассчитано общее количество НЯ/СНЯ, количество пациентов с НЯ/СНЯ (процент от общего числа пациентов, включенных в популяцию анализа безопасности). Была проанализирована тяжесть НЯ и связь с приемом препарата исследуемой терапии (по мнению врача). Отдельно представлена информация о частоте НЯ/СНЯ, расце- ненных исследователем как связанных с применением препаратов. Изменение лабораторных показателей, выходящее за границы нормальных значений, и их нормализация представлены в виде частотных таблиц для всех клинических показателей. Для всех показателей безопасности проводились межгрупповые сравнения. Для сравнения частоты возникновения НЯ в группах наблюдения применялся критерий $X^{2}$, дисперсионный анализ ANOVA (при условии нормального распределения и равенстве дисперсий) или соответствующий непараметрический аналог (ранговый критерий Крускала-Уоллиса для оценки разностей между медианами с поправкой Бонферрони для множественных сравнений).

Связь НЯ с исследуемым препаратом оценивалась следующим образом: нет связи, сомнительная, возможная, вероятная, определенная.

\section{РЕЗУЛЬТАТЫ}

В исследовании приняли участие 150 пациентов. В таблице 3 представлены демографические и антропометрические данные по группам пациентов.

Значимых различий в возрасте, массе тела, росте, ИМТ и в распределении по полу между группами не выявлено.

У 96 пациентов (64\%), включенных в исследование, были выявлены сопутствующие заболевания, среди которых наиболее распространенными оказались гипертоническая болезнь - 12,6\% (24/191) и остеохондроз позвоночника - 11\% (21/191). Все хронические заболевания находились вне стадии обострения.

Все отклонения лабораторных показателей от нормальных значений на этапе скрининга у пациентов были расценены врачами-исследователями как клинически не значимые.

\section{Оценка эффективности по первичной конечной точке}

В соответствии с протоколом исследования необходимо было оценить величину изменения концентрации 25OHD в крови пациентов к Визиту 3 относительно Визита 1.

При оценке уровня 25OHD в крови пациентов групп T, R и X к Визиту 3 относительно Визита 1 в группе Т среднее значение величины изменения концентрации

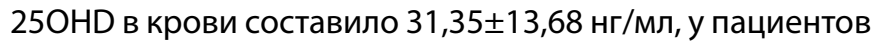
группы $R-29,59 \pm 12,22$ нг/мл, у пациентов группы X $13,87 \pm 7,49$ нг/мл (рис. 1).

Согласно протоколу, доказательством гипотезы превосходства является установление между группами статистически значимой разницы по величине прироста

Таблица 3. Демографические и антропометрические показатели исследуемых лиц по группам лечения

\begin{tabular}{|c|c|c|c|}
\hline Группы/показатели & 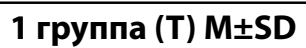 & 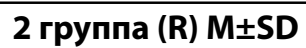 & 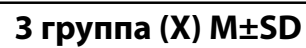 \\
\hline Соотношение м/ж & $3 / 47$ & $6 / 44$ & $5 / 45$ \\
\hline Возраст, годы & $42,04 \pm 15,38$ & $43,06 \pm 16,15$ & $43,68 \pm 14,41$ \\
\hline Рост, см & $166,47 \pm 6,79$ & $166,18 \pm 6,58$ & $166,18 \pm 6,58$ \\
\hline Вес, кг & $69,13 \pm 10,62$ & $69,82 \pm 9,86$ & $69,82 \pm 9,86$ \\
\hline 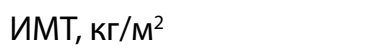 & $24,91 \pm 3,3$ & $25,29 \pm 3,32$ & $25,29 \pm 3,32$ \\
\hline
\end{tabular}




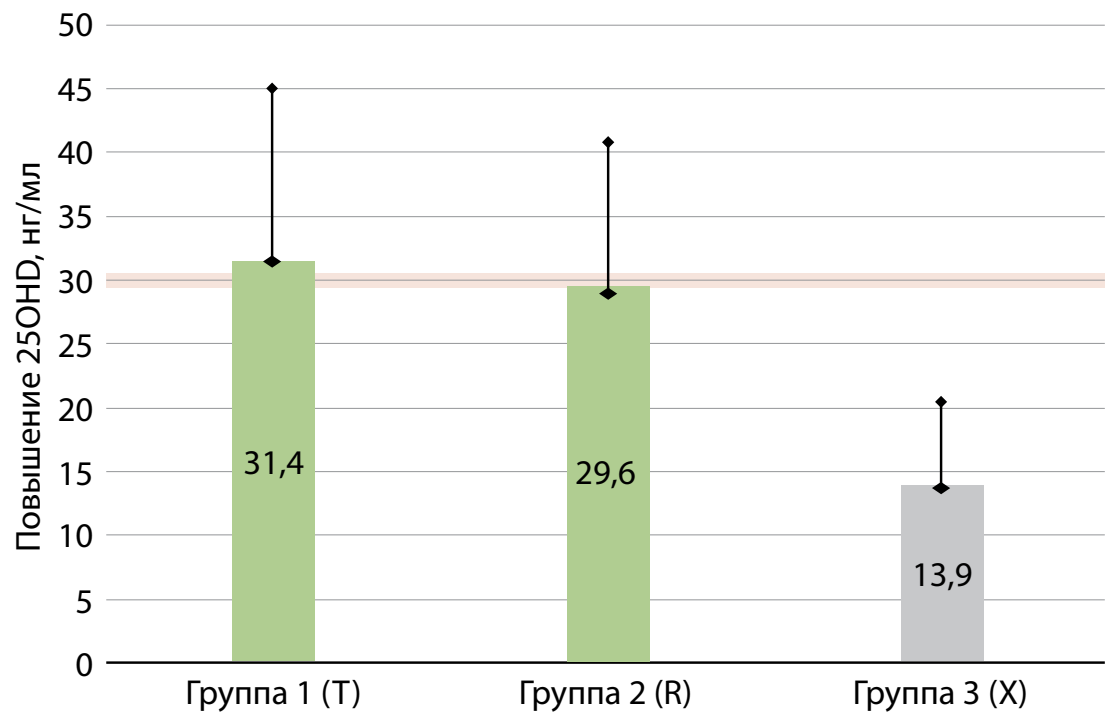

Рис. 1. Изменение концентрации 25OHD за 2 мес лечения дефицита витамина D по группам терапии.

концентрации 25OHD в крови пациентов к Визиту 3. То есть установление величины $\mathrm{SM} \geq 3,197$ нг/мл, соответствующей клинически значимой разнице между группами пациентов с дефицитом витамина D.

Различия между величиной изменения основного критерия эффективности в группах составили:

1) между группой Фортедетрим в дозовом режиме 50000 ME 1 раз в неделю и группой Вигантол ${ }^{\circledR}$ по 2 капли 1000 ME в день разница по величине прироста концентрации 25OHD в крови пациентов к Визиту 3 составила 17,48 нг/мл и $95 \%$ ДИ $(13,07 \div 21,89)$;

2) между группой Фортедетрим в дозовом режиме 8000 ME ежедневно и группой Вигантол ${ }^{\circledR}$ по 2 капли $1000 \mathrm{ME}$ в день разница по величине прироста концентрации 25OHD в крови пациентов к Визиту 3 составила 15,72 нг/мл и 95\% ДИ $(11,68 \div 19,76)$. Таким образом, нижняя граница 95\% ДИ для разницы по величине прироста концентрации 25OHD в крови пациентов к Визиту 3 в обоих случаях превысила установленную протоколом границу превосходства

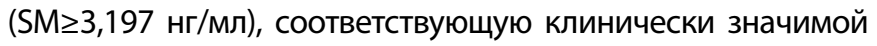
разнице между группами пациентов с дефицитом витамина $D$, следовательно, гипотезу о превосходстве терапии препаратом Фортедетрим в обоих дозовых режимах (50 000 и 56000 МЕ в неделю) над терапией препаратом Вигантол ${ }^{\circledR}$ (7000 ME в неделю) можно считать доказанной.

\section{Оценка эффективности по вторичным конечным точкам}

Динамика концентрации 25OHD с 1 по 3 визиты (Этап I)

В соответствии с протоколом исследования необходимо было определить динамику концентрации 25OHD с 1 по 3 визиты (Этап I).
У пациентов группы Т среднее значение концентрации

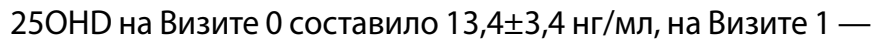
$15,5 \pm 6,2$ нг/мл, на Визите 2 - 37,2 \pm 11 нг/мл, на Визите 3 -

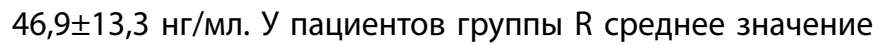
концентрации 25OHD на Визите 0 составило 14,3 2,9 нг/мл, на Визите $1-15,6 \pm 5,6$ нг/мл, на Визите $2-36,2 \pm 9,7$ нг/мл, наВизите 3-45,2ะ12,7 нг/мл.УпациентовгруппыХсреднее значение концентрации 25OHD на Визите 0 составило $14,5 \pm 3,5$ нг/мл, на Визите $1-14,9 \pm 3,6$ нг/мл, на Визите 2 $23,3 \pm 5,9$ нг/мл, на Визите $3-28,6 \pm 8,2$ нг/мл (см. рис. 2 и 3 )

В результате сравнительного анализа средних значений концентраций 25OHD у пациентов групп T, R и X с 1 по 3 Визиты (Этап I) были выявлены значимые различия между группами Т и X и между группами R и X на Визитах 2 и 3 ( $<<0,0001)$ для всех различий.

На рисунке 4 представлены доли пациентов в каждой группе, которые достигли к визитам 2 и 3 повышения концентрации 25OHD более 20 нг/мл (справа) и более 30 нг/мл (слева). Как видно из рис. 4, практически все пациенты 1 и 2 групп (98\%) к визиту 3 достигли концентрации более 20 нг/мл и у 90\% группы сравнения и значимых различий к визиту 3 не наблюдалось. Что касается уровня 25OHD более 30 нг/мл, то к визиту 3 в группах 1 и 2 этого уровня достигли соответственно 90 и 88\% пациентов, а в группе сравнения - только 42\%. Различия между группами 1 и 3 и между группами 2 и 3 были статистически значимы.

В таблице 4 суммированы итоги лечения к визитам 4 и 6. K 4 визиту в группах лечения были достигнуты средние уровни витамина D 43-45 нг/мл, в то время как в группе сравнения этот показатель составил 29,94нг/мл. K 6 визиту содержание витамина D в крови у участников 1 и 2 групп было соответственно 39-35 нг/мл против

Таблица 4. Итоги 2 этапа по достижению концентрации 25OHD более 30 нг/мл

\begin{tabular}{lcccc}
\hline \multirow{2}{*}{ Группы } & \multicolumn{2}{c}{ 4 визит } & \multicolumn{2}{c}{ визит } \\
\cline { 2 - 5 } & Уровень витамина D & $\begin{array}{c}\text { Доля в \% } \\
\text { >30 нг/мл }\end{array}$ & Уровень витамина D & $\begin{array}{c}\text { Доля в \% } \\
>\mathbf{3 0} \text { нг/мл }\end{array}$ \\
\hline Группа 1 (T) & $44,95 \pm 11,8$ & 96 & $38,93 \pm 12,4$ & 68 \\
Группа 2 (R) & $43,11 \pm 8,8$ & 94 & $35,01 \pm 7,92$ & 48 \\
Группа 3 (X) & $29,94 \pm 8,09$ & 48 & $27,35 \pm 8,03$ & 36 \\
\hline
\end{tabular}


Группа 1 (Т) 50000 ME 1 раз в неделю

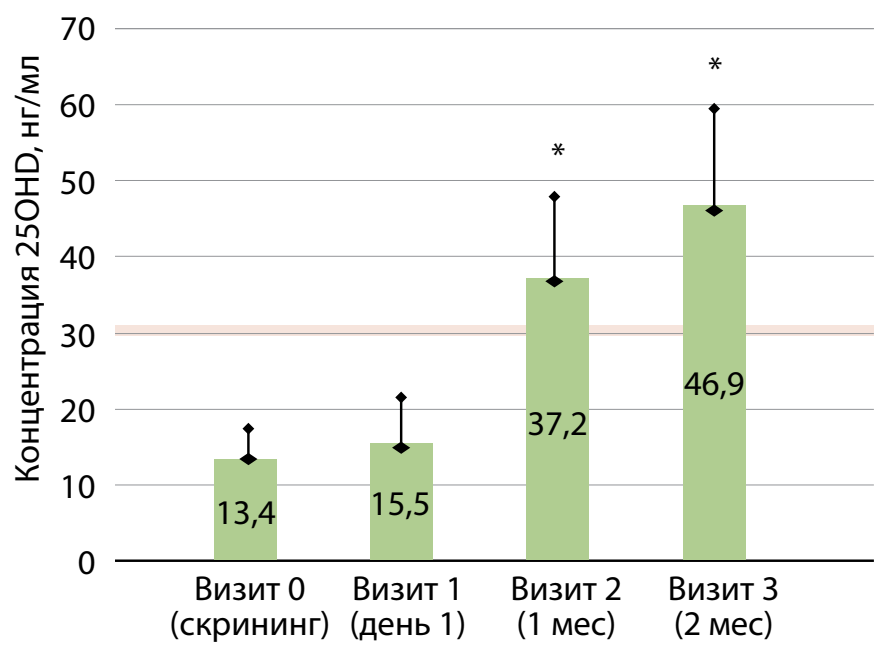

Группа 3 (X) 1000 ME ежедневно

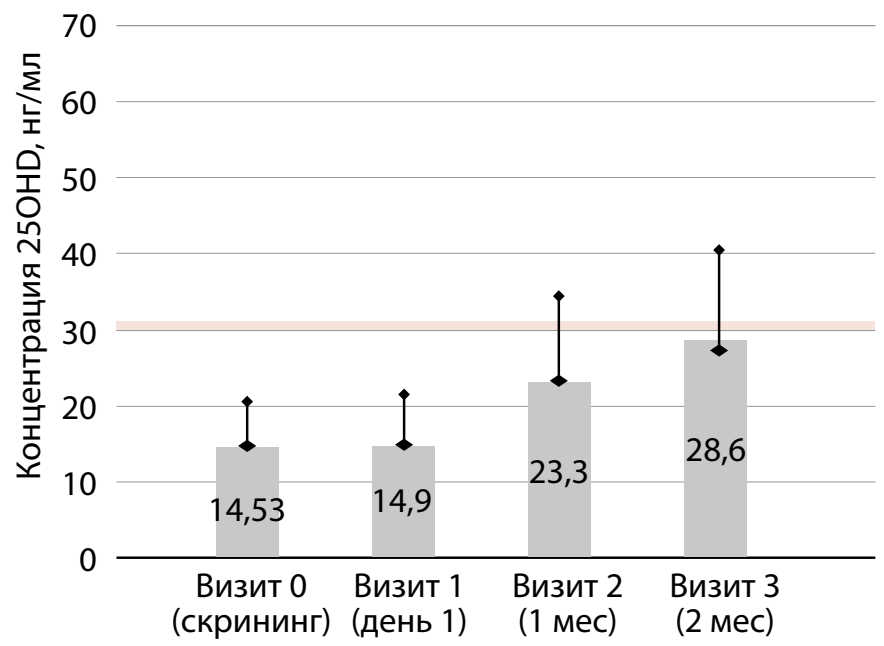

* - p $<0,0001$ для группа 1 vs группа 3.

Рис. 2. Динамика содержания 25OHD в крови по визитам в 1 группе (T) в сравнении с группой 3 (X).

Группа 2 (R) 8000 ME ежедневно

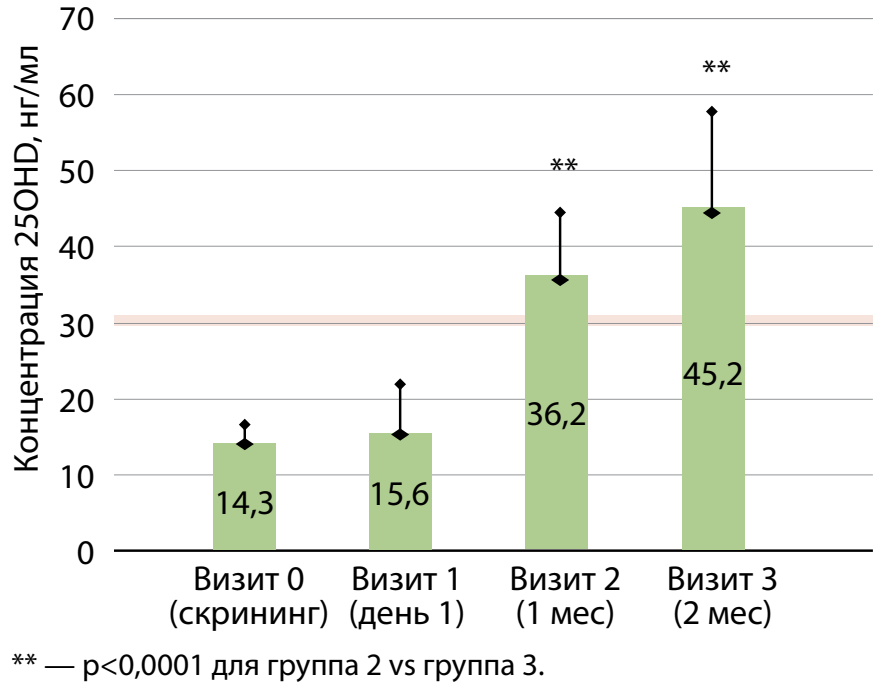

Группа 3 (X) 1000 ME ежедневно

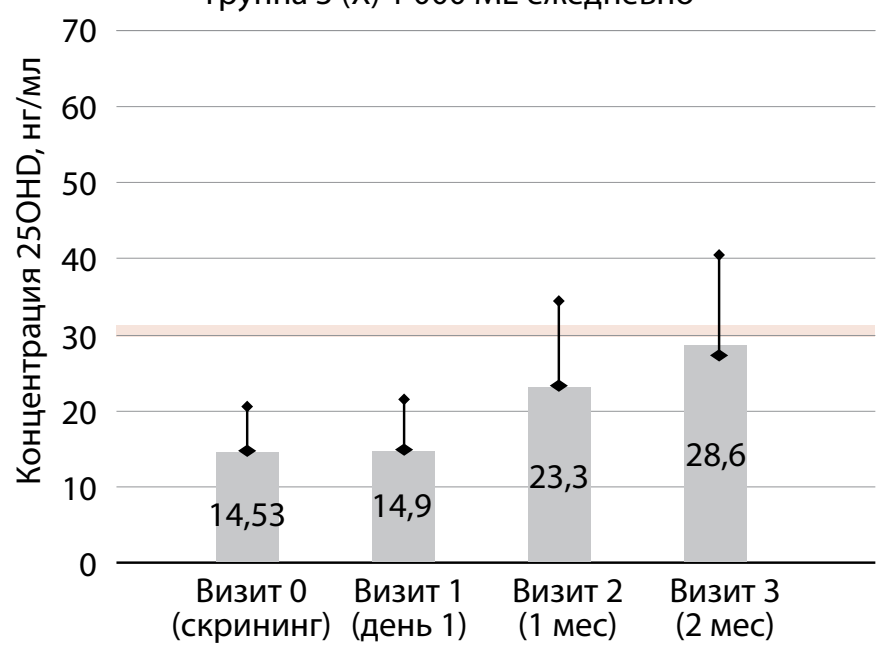

Рис. 3. Динамика содержания $25 \mathrm{OHD}$ в крови по визитам в 2 группе (R) в сравнении с группой 3 (X).

$>20$ нг/мл

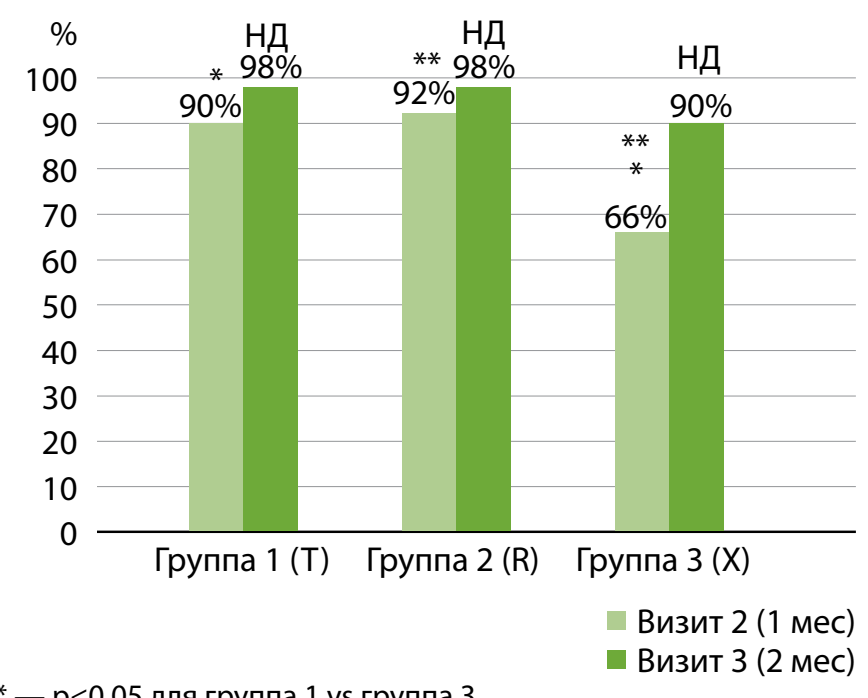

$>30 \mathrm{Hг} / \mathrm{Mr}$

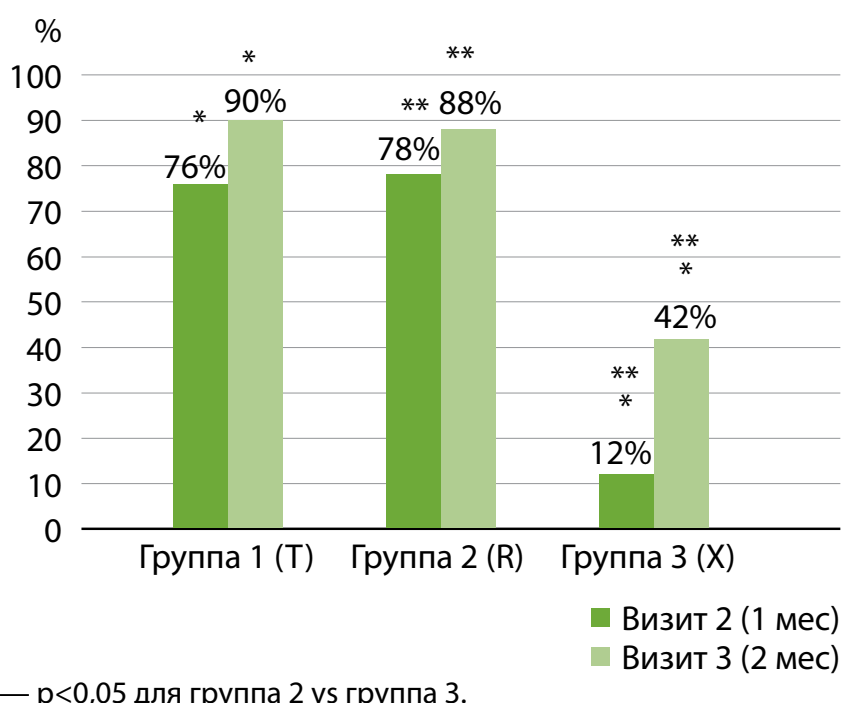

Рис. 4. Доли пациентов в \%, которые достигли к 3 визиту концентраций витамина D более 20 и 30 нг/мл. 
27,35 нг/мл в группе сравнения. В таблице 4 представлены доли пациентов в каждой группе, достигших уровня $250 H D$ более 30 нг/мл.

\section{ОЦЕНКА БЕЗОПАСНОСТИ}

Оценку безопасности проводили для всех 150 пациентов, включенных в исследование, на статистическом анализе конечных точек безопасности.

Доля пациентов с зарегистрированными случаями НЯ в течение всего периода исследования составила 28,00\% (42/150). Всего у 42 пациентов отмечалось 83 НЯ. В течение исследования у пациентов всей популяции отмечалось 92,77\% (77/83) НЯ легкой степени выраженности и 7,23\% (6/83) НЯ умеренной степени выраженности. По оценке врачей-исследователей, причинно-следственная связь с терапией исследуемым препаратом/препаратом сравнения отсутствовала в 66,27\% (55/83) случаях, была расценена как сомнительная в 20,48\% (17/83) случаях, как возможная - в 8,43\% (7/83) случаях, как вероятная - в 4,82\% (4/83) случаях. Анализ частоты исходов НЯ у пациентов показал, что к завершению исследования «выздоровление без последствий» отмечалось в 95,18\% (79/83) случаях, состояние без изменений отмечалось в 4,82\% (4/83) случаях.

Доля пациентов группы Т (1) с зарегистрированными случаями НЯ в течение всего периода исследования составила 30,00\% (15/50). Всего у 15 пациентов этой группы отмечалось 26 НЯ. В течение исследования у пациентов группы Т отмечалось 96,15\% (25/26) НЯ легкой степени выраженности и 3,85\% (1/26) НЯ умеренной степени выраженности. По оценке врачей-исследователей, причинно-следственная связь НЯ с терапией исследуемым препаратом у пациентов группы Т отсутствовала в $80,77 \%(21 / 26)$ случаях, расценивалась как сомнительная в 19,23\% (5/26) случаях. Анализ частоты исходов НЯ у пациентов группы Т показал, что к завершению исследования «выздоровление без последствий» отмечалось в $100,00 \%(26 / 26)$ случаях.

Доля пациентов группы R(2) с зарегистрированными случаями НЯ в течение всего периода исследования составила 22,00\% (11/50). Всего у 11 пациентов группы $\mathrm{R}$ отмечалось 24 НЯ. В течение исследования у пациентов группы R отмечалось 91,67\% (22/24) НЯ легкой степени выраженности и 8,33\% (2/24) НЯ умеренной степени выраженности. По оценке врачей-исследователей, причинно-следственная связь НЯ с терапией исследуемым препаратом у пациентов группы R отсутствовала в 62,50\% (15/24) случаях, была расценена как сомнительная в 16,67\% (4/24) случаев, как возможная - в 4,17\% (1/24) случаев, как вероятная - в 16,67\% (4/24) случаев. Анализ частоты исходов НЯ у пациентов группы R показал, что к завершению исследования «выздоровление без последствий» отмечалось в 91,67\% $(22 / 24)$ случаях, состояние без изменений отмечалось в 8,33\% (2/24) случаях.

Доля пациентов группы X с зарегистрированными случаями НЯ в течение всего периода исследования составила 32,00\% (16/50). Всего у 16 пациентов группы X отмечалось 33 НЯ. В течение исследования у пациентов группы X отмечалось 90,91\% (30/33) НЯ легкой степени выраженности и 9,09\% (3/33) НЯ умеренной степени выраженности. По оценке врачей-исследователей, причинно-следственная связь НЯ с терапией исследуемым препаратом у пациентов группы Х отсутствовала в 57,58\% (19/33) случаях, была расценена как сомнительная в 24,24\% (8/33) случаев, как возможная - в 18,18\% (6/33) случаев. Анализ частоты исходов НЯ у пациентов группы $\mathrm{X}$ показал, что к завершению исследования «выздоровление без последствий» отмечалось в 93,94\% (31/33) случаях, состояние без изменений отмечалось в 6,06\% (2/33) случаях.

В ходе исследования летальных случаев, прочих СНЯ и других значимых НЯ зарегистрировано не было.

В результате сравнительного анализа НЯ по их наличию не было выявлено межгрупповых различий. В результате сравнительного анализа НЯ по степени выраженности НЯ, связи с исследуемым препаратом/препаратом сравнения и по исходу НЯ у пациентов в группах T, R и X с зарегистрированными НЯ не было выявлено межгрупповых различий.

Как видно из таблицы 5, в процессе лечения дефицита витамина D препаратами колекальциферола в разных дозировках значимых изменений ПТГ, креатинина, кальция крови и мочи, фосфатов не обнаружено. Также случаев гиперкальциемии и гиперкальциурии ни в одной из групп не выявлено.

Не обнаружено отрицательной динамики по результатам оценки витальных функций, общего и биохимического анализов крови, общего анализа мочи. Выявленные отклонения от нормальных значений лабораторных

Таблица 5. Динамика показателей кальций-фосфорного обмена и креатинина исходно и 6 визиту

\begin{tabular}{|c|c|c|c|c|c|c|}
\hline \multirow{2}{*}{$\begin{array}{l}\text { Показатели/ } \\
\text { группы }\end{array}$} & \multicolumn{2}{|c|}{ Группа 1 (T) } & \multicolumn{2}{|c|}{ Группа 2 (R) } & \multicolumn{2}{|c|}{ Группа 3 (X) } \\
\hline & Визит 0 & Визит 6 & Визит 0 & Визит 6 & Визит 0 & Визит 6 \\
\hline $\begin{array}{l}\text { Паратгормон } \\
\text { (15-65 пг/мл) }\end{array}$ & 35,17 & 30,74 & 35,29 & 27,6 & 36,56 & 29,6 \\
\hline $\begin{array}{l}\text { Кальций общий в крови } \\
(2,15-2,55 \text { ммоль/л) }\end{array}$ & 2,32 & 2,38 & 2,34 & 2,39 & 2,34 & 2,36 \\
\hline $\begin{array}{l}\text { Кальций в моче } \\
(2,5-7,5 \text { ммоль/сут) }\end{array}$ & 3,84 & 4,31 & 2,68 & 3,79 & 3,08 & 2,84 \\
\hline $\begin{array}{l}\text { Фосфор } \\
(0,81-1,45 \text { ммоль/л) }\end{array}$ & 1,17 & 1,22 & 1,15 & 1,22 & 1,18 & 1,29 \\
\hline $\begin{array}{l}\text { Креатинин } \\
\text { (55-88 мкмоль/л) }\end{array}$ & 73,2 & 72,23 & 76,1 & 73,17 & 72,8 & 71,5 \\
\hline
\end{tabular}


показателей были расценены врачами-исследователями как клинически незначимые.

\section{ОБСУЖДЕНИЕ РЕЗУЛЬТАТОВ}

Витамин D относится к группе жирорастворимых витаминов. Он естественным образом присутствует лишь в очень ограниченном количестве продуктов питания, а синтез в организме человека возможен только в определенных условиях, когда ультрафиолетовые лучи солнечного света попадают на кожу. Витамин $D$, получаемый из продуктов питания и в виде пищевых добавок (эргокальциферол - витамин $\mathrm{D}_{2}$ ), а также образующийся в коже (колекальциферол - витамин $\mathrm{D}_{3}$ ) при пребывании на солнце, биологически инертен. Для активации и превращения в активную форму D-гормона в организме должны пройти два процесса гидроксилирования [1]. 1 этап - гидроксилирование в печени при помощи 25-гидроксилаз с образованием транспортной формы 25OHD - кальцидиола; 2 этап - гидроксилирование преимущественно в почках с помощью 1а-гидроксилазы с образованием 1,25(OH) $\mathrm{D}$ активного метаболита, непосредственно связывающегося в рецептором витамина D и оказывающим свое многообразное действие на различные органы и ткани [2].

Витамин D способствует абсорбции кальция в кишечнике и поддерживает необходимые уровни кальция и фосфатов в крови для обеспечения минерализации костной ткани и предотвращения гипокальциемической тетании. Он также необходим для роста костей и процесса костного ремоделирования, т.е. работы остеобластов и остеокластов. Достаточный уровень витамина D предотвращает развитие рахита у детей и остеомаляции у взрослых. Вместе с кальцием витамин D также применяется для профилактики и в составе комплексного лечения остеопороза $[3,5]$

Согласно мнению ряда исследователей, функции витамина D не ограничены только контролем кальций-фосфорного обмена, он также влияет и на другие физиологические процессы в организме, включающие модуляцию клеточного роста, нервно-мышечную проводимость, иммунитет и воспаление [13]. Экспрессия большого количества генов, кодирующих белки, участвующие в пролиферации, дифференцировке и апоптозе, регулируется витамином D. Многие клетки имеют рецепторы к витамину D, а в некоторых тканях присутствует собственная 1а-гидроксилаза для образования активной формы D-гормона, и они могут локально генерировать его высокие внутриклеточные концентрации для своих собственных целей функционирования без увеличения его концентрации в общем кровотоке [14].

Ввиду широкого распространения недостаточности и дефицита витамина D как во всем мире [15], так и в России [16] имеется настоятельная необходимость в наличии широкого спектра медицинских препаратов витамина D (колекальциферола) в разных дозировках и лекарственных формах для адекватного применения в насыщающих и поддерживающих дозах для лечения дефицита витамина D.

На сегодняшний день препарат Аквадетрим, капли для приема внутрь (в 1 капле $500 \mathrm{ME}$ колекальциферола) зарегистрирован на территории РФ и широко применяется в профилактике и лечении дефицита витамина D, так же как и Вигантол ${ }^{\circledR}$ капли для приема внутрь (в 1 капле $500 \mathrm{ME}$ колекальциферола). Но эти препараты более удобны для профилактики дефицита витамина D у взрослых и рахита у детей, но не для применения в насыщающих и поддерживающих доз для лечения.

Компанией «МЕДАНА ФАРМА» АО, Польша с целью регистрации препаратов Фортедетрим капсулы мягкие 4000 и 10000 МЕ («МЕДАНА ФАРМА»АО, Польша) на территории РФ в соответствии с Федеральным законом №61-Ф3 от 12.04.2010 г. «Об обращении лекарственных средств» было проведено данное клиническое исследование: «Открытое многоцентровое сравнительное рандомизированное исследование эффективности и безопасности препарата Фортедетрим, капсулы мягкие («МЕДАНА ФАРМА» АО, Польша) при использовании насыщающих и поддерживающих доз в сравнении с терапией препаратом Вигантол ${ }^{\circledR}$, раствор для приема внутрь масляный («Мерк КГаА», Германия) у пациентов с дефицитом витамина D» Способ применения, режим дозирования и длительность терапии исследуемым препаратом были выбраны в соответствии с клиническими рекомендациями [17], доступной научной информацией, а также с учетом результатов клинических исследований [18-24]. Способ применения, режим дозирования и длительность терапии препаратом сравнения были выбраны в соответствии с инструкцией по медицинскому применению препарата Вигантол ${ }^{\circledR}$.

В соответствии с клиническими рекомендациями Российский ассоциации эндокринологов (РАЭ) [4] при дефиците витамина D применяется методика насыщающих и поддерживающих доз с возможной разной кратностью приема (табл. 6), что было использовано в нашем исследовании.

Целью нашего исследования была оценка эффективности и безопасности терапии препаратом Фортедетрим, капсулы мягкие («МЕДАНА ФАРМА» АО, Польша) при использовании насыщающих и поддерживающих доз в сравнении с терапией препаратом Вигантол ${ }^{\circledR}$, раствор для приема внутрь масляный («Мерк КГаА», Германия) у пациентов с дефицитом витамина D.

Таким образом для насыщающих доз групп 1(T) и 2(R) использовали капсулы Фортедетрим соответственно $50000 \mathrm{ME} 1$ раз в неделю (5 капсул по $10000 \mathrm{ME}$ ) и 56000 ME-8000 ME ежедневно (2 капсулы по 4000 ME), а в группе сравнения $3(\mathrm{X})$ - Вигантол ${ }^{\circledR}$ по $1000 \mathrm{ME}$ ежедневно (2 капли); для поддерживающих доз (2 этап исследования) для группы 1 применяли Фортедетрим 10000 ME 1 раз в неделю, а для группы 2 по 2000 МЕ ежедневно (14000 МЕ в неделю); в группе сравнения продолжали прием Вигантола 1000 МЕ ежедневно.

Как видно из результатов исследования в группах 1 и 2 на всех визитах и этапах исследования как концентрация 25OHD, так и величина его изменения, и доля пациентов, достигших уровня 25OHD в крови более 30 нг/мл, были достоверно выше на 3-6 визитах, чем в группе сравнения, что подтверждает выполнение первичной и вторичных точек эффективности. Тем не менее необходимо отметить, что и длительный прием колекальциферола в дозе 1000 ME ежедневно к 20 неделям (6 визиту) позволил достичь уровня 30 нг/мл у $36 \%$ участников, но это значимо меньше, чем 1 и 2 группах с применением высокодозных препаратов. 
Таблица 6. Схемы лечения дефицита витамина D

Доза колекальциферола

Примеры схем лечения*

Коррекция дефицита витамина D (при уровне $250 \mathrm{HD}<20$ нг/мл) - насыщающие дозы

150000 MЕ еженедельно в течение 8 нед внутрь

2200000 ME ежемесячно в течение 2 мес внутрь

3150000 ME ежемесячно в течение 3 мес внутрь

47000 МЕ в день - 8 нед внутрь
Аквадетрим или Вигантол ${ }^{\circledR}: 100$ капель в неделю или по 50 капель 2 раза в неделю

Вигантол ${ }^{\circledR}: 10$ мл (1 флакон) внутрь 1 раз в месяц 2 мес

Аквадетрим: 10 мл (1 флакон) внутрь 1 раз в месяц 3 мес

Аквадетрим или Вигантол ${ }^{\circledR} 14$ капель в день - 8 нед

Поддержание уровней витамина D >30 нг/мл - поддерживающие дозы

1 1000-2000 МЕ ежедневно внутрь

\section{6000-14 000 МЕ однократно в неделю внутрь}

Что касается безопасности лечения дефицита витамина D колекальциферолом (Фортедетримом) в максимальных дозах 8000 ME ежедневно или 50000 МЕ однократно 1 раз в неделю, то различий в количестве НЯ, их степени, связи с приемом препаратов между группами лечения и группой сравнения не выявлено, что соответствует данным литературы о безопасности применения колекальциферола до 10000 МЕ ежедневно [12]

\section{ЗАКЛЮЧЕНИЕ}

Таким образом, в результате оценки данных по результатам I и II этапов проведенного клинического исследования Фортедетрима в сравнении с Вигантолом ${ }^{\circledR}$ была доказана гипотеза о превосходстве высокодозного препарата Фортедетрим (капсулы по 4000 и 10000 МЕ) над препаратом Вигантол ${ }^{\circledR}$ (капли 1000 МЕ ежедневно), с эквивалентной безопасностью лечения. Тем не менее необходим индивидуальный подход к восполнению дефицита витамина D, так как не все пациенты в примененных режимах насыщения достигли целевых значений 25OHD в крови, с учетом комплаентности, степени ожирения, нарушений всасываемости Са и витамина D.

\section{ДОПОЛНИТЕЛЬНАЯ ИНФОРМАЦИЯ}

Источники финансирования. Исследование выполнено при финансовом и лекарственном обеспечении «МЕДАНА ФАРМА» АО, Польша.

Конфликт интересов. Авторы декларируют отсутствие явных и потенциальных конфликтов интересов, связанных с содержанием настоящей статьи.

\author{
2-4 капли в сутки \\ Аквадетрим/Вигантол ${ }^{\circledR}$ 15-30 капель однократно \\ в неделю
}

Участие авторов. Л.Я. Рожинская - существенный вклад в концепцию и дизайн исследования, в получение, анализ данных или интерпретацию результатов, написание статьи; Е.А. Пигарова существенный вклад в концепцию и дизайн исследования, в получение, анализ данных или интерпретацию результатов, написание статьи; А.А. Багрецова - существенный вклад в получение, анализ данных или интерпретацию результатов, внесение в рукопись существенной правки с целью повышения научной ценности статьи; А.Ф. Вербовой - существенный вклад в получение, анализ данных или интерпретацию результатов, внесение в рукопись существенной правки с целью повышения научной ценности статьи; Н.Г. Кондратьева - существенный вклад в получение, анализ данных или интерпретацию результатов, внесение в рукопись существенной правки с целью повышения научной ценности статьи; О.А. Василевская - существенный вклад в получение, анализ данных или интерпретацию результатов, внесение в рукопись существенной правки с целью повышения научной ценности статьи; В.Б. Василюк - существенный вклад в получение, анализ данных или интерпретацию результатов, внесение в рукопись существенной правки с целью повышения научной ценности статьи; М.В. Манько - существенный вклад в получение, анализ данных или интерпретацию результатов, внесение в рукопись существенной правки с целью повышения научной ценности статьи; В.Б. Шуньков - существенный вклад в получение, анализ данных или интерпретацию результатов, внесение в рукопись существенной правки с целью повышения научной ценности статьи; Т.А. Гребенникова - существенный вклад в получение, анализ данных или интерпретацию результатов, внесение в рукопись существенной правки с целью повышения научной ценности статьи.

Все авторы одобрили финальную версию статьи перед публикацией, выразили согласие нести ответственность за все аспекты работы, подразумевающую надлежащее изучение и решение вопросов, связанных с точностью или добросовестностью любой части работы.

\section{СПИСОК ЛИТЕРАТУРЫ | REFERENCES}

1. Holick MF. Vitamin D Deficiency. N. Engl. J. Med. 2007;357(3):266-281. doi: https://doi.org/10.1056/NEJMra070553.

2. Scragg R, Waayer D, Stewart AW, et al. The Vitamin D Assessment (ViDA) Study: design of a randomized controlled trial of vitamin D supplementation for the prevention of cardiovascular disease, acute respiratory infection, falls and non-vertebral fractures. The Journal of Steroid Biochemistry and Molecular Biology. 2016;164:318-325. doi: https://doi.org/10.1016/j.jsbmb.2015.09.010.

3. Institute of Medicine (US) Committee to Review Dietary Reference Intakes for Vitamin D and Calcium; Ross AC, Taylor CL, Yaktine AL, et al., editors. Dietary Reference Intakes for Calcium and Vitamin D. Washington (DC): National Academies Press (US); 2011 Available from: https://www.ncbi.nlm.nih.gov/books/NBK56070/ doi: https://doi.org/10.17226/13050 
4. Пигарова Е.А., Рожинская Л.Я, Белая Ж.Е. и др. Клинические рекомендации Российской ассоциации эндокринологов по диагностике, лечению и профилактике дефицита витамина D у взрослых // Проблемы эндокринологии. - 2016 - Т. 62. №4. - C. 60-84. [Pigarova E.A., Rozhinskaya L.Y., Belaya J.E., Dzeranova LK, Karonova TL, Ilyin AV, Melnichenko GA, Dedov II. Russian Association of Endocrinologists recommendations for diagnosis, treatment and prevention of vitamin D deficiency in adults. Problems of Endocrinology. 2016;62(4):60-84. (In Russ.)]. doi: https://doi.org/10.14341/probl201662460-84

5. Cranney A, Horsley T, O'Donnell S, et al. Effectiveness and safety of vitamin D in relation to bone health. 2007 In: Database of Abstracts of Reviews of Effects (DARE): Quality-assessed Reviews [Internet]. York (UK): Centre for Reviews and Dissemination (UK); 1995-. Available from: https://www.ncbi.n/m.nih.gov/books/NBK74011/

6. Scragg R, Waayer D, Stewart AW, et al. The Vitamin D Assessment (ViDA) Study: design of a randomized controlled trial of vitamin D supplementation for the prevention of cardiovascular disease, acute respiratory infection, falls and non-vertebral fractures. The Journal of Steroid Biochemistry and Molecular Biology. 2016;164:318-325. doi: https://doi.org/10.1016/j.jsbmb.2015.09.010.

7. Scragg R, Khaw K-T, Toop L, et al. Monthly HighDose Vitamin D Supplementation and Cancer Risk. JAMA Oncology. 2018;4(11):e182178. doi: https://doi.org/10.1001/jamaoncol.2018.2178.

8. Soe HHK, Abas ABL, Than NN, et al. Vitamin D supplementation for sickle cell disease. Cochrane Database Syst Rev. 2017. doi: https://doi.org/10.1002/14651858.CD010858.pub2

9. Schleck M-L, Souberbielle J-C, Jandrain B, et al. A Randomized, Double-Blind, Parallel Study to Evaluate the Dose-Response of Three Different Vitamin D Treatment Schemes on the 25-Hydroxyvitamin D Serum Concentration in Patients with Vitamin D Deficiency. Nutrients. 2015;7(7):5413-5422. doi: https://doi.org/10.3390/nu7075227.

10. Ilahi M, Armas LAG, Heaney RP. Pharmacokinetics of a single, large dose of cholecalciferol. The American Journal of Clinical Nutrition. 2008;87(3):688-691. doi: https://doi.org/10.1093/ajcn/87.3.688.

11. Dudenkov DV, Yawn BP, Oberhelman SS, et al. Changing Incidence of Serum 25-Hydroxyvitamin D Values Above $50 \mathrm{ng} / \mathrm{mL}$ : A 10-Year Population-Based Study. Mayo Clin. Proc. 2015;90(5):577-586. doi: https://doi.org/10.1016/..mayocp.2015.02.012.

12. Cannell JJ, Hollis BW. Use of vitamin D in clinical practice. Altern. Med. Rev. 2008;13(1):6-20.

13. Quack Lötscher KC, I'Allemand D, Bischoff-Ferrari HA, Burckhardt P. Vitamin-D deficiency: Evidence, safety, and recommendations for the Swiss population. Bern, Switzerland: Federal Office of Public Health, 2012

14. Holick MF, Binkley NC, Bischoff-Ferrari HA, et al. Evaluation, Treatment, and Prevention of Vitamin D Deficiency: an Endocrine Society Clinical
Practice Guideline. J. Clin. Endocr. Metab. 2011:96(7):1911-1930.

doi: https://doi.org/10.1210/jc.2011-0385

15. Holick MF, Chen TC. Vitamin D deficiency: a worldwide problem with health consequences. The American Journal of Clinical Nutrition. 2008;87(4):1080S-1086S. doi: https://doi.org/10.1093/ajcn/87.4.1080S.

16. Петрушкина А.А., Пигарова Е.А., Рожинская Л.Я Эпидемиология дефицита витамина D в российской федерации // Остеопороз и остеопатии. - 2018. - Т. 21. №3. - C. 15-20. [Petrushkina AA, Pigarova EA, Rozhinskaya LY. The prevalence of vitamin D deficiency in Russian Federation. Osteoporosis and Bone Diseases. 2019;21(3):15-20. (In Russ.)] doi: https://doi.org/10.14341/osteo10038

17. Пигарова Е.А. Основные положения клинических рекомендаций Российской Ассоциации эндокринологов «Дефицит витамина D у взрослых: диагностика, лечение и профилактика» // Остеопороз и остеопатии. - 2015. T. 18. - №2. - C. 29-32. [Pigarova E.A. Summary of clinical guidelines of the Russian association of endocrinologists vitamin D deficiency in adults: diagnosis, treatment and prevention. Osteoporosis and Bone Diseases. 2015;18(2):29-32. (In Russ.)] doi: https://doi.org/10.14341/osteo2015229-32

18. Bischoff-Ferrari HA, Willett WC, Wong JB, et al. Fracture Prevention With Vitamin D Supplementation. JAMA. 2005;293(18):2257. doi: https://doi.org/10.1001/jama.293.18.2257.

19. Gagnon C, Lu ZX, Magliano DJ, et al. Serum 25-hydroxyvitamin D, calcium intake, and risk of type 2 diabetes after 5 years: results from a national, population-based prospective study (the Australian Diabetes, Obesity and Lifestyle study). Diabetes Care. 2011;34(5):1133-1138. doi: https://doi.org/10.2337/dc10-2167.

20. Parker J, Hashmi O, Dutton D, et al. Levels of vitamin D and cardiometabolic disorders: Systematic review and meta-analysis. Maturitas. 2010;65(3):225-236 doi: https://doi.org/10.1016/j.maturitas.2009.12.013.

21. Drechsler C, Pilz S, Obermayer-Pietsch B, et al. Vitamin D deficiency is associated with sudden cardiac death, combined cardiovascular events, and mortality in haemodialysis patients. Eur. Heart J. 2010;31(18):2253-2261. doi: https://doi.org/10.1093/eurheartj/ehq246.

22. Jackson C, Gaugris S, Sen SS, Hosking D. The effect of cholecalciferol (vitamin D3) on the risk of fall and fracture: a meta-analysis. QJM. 2007;100(4):185-192. doi: https://doi.org/10.1093/qjmed/hcm005.

23. Schnatz $P F$, Jiang $X$, Vila-Wright $S$, et al. Calcium/vitamin D supplementation, serum 25-hydroxyvitamin D concentrations, and cholesterol profiles in the Women's Health Initiative calcium/ vitamin D randomized trial. Menopause. 2014;21(8):823-833. doi: https://doi.org/10.1097/gme.0000000000000188.

24. Garland CF, Gorham ED, Mohr SB, Garland FC. Vitamin D for Cancer Prevention: Global Perspective. Ann. Epidemiol. 2009;19(7):468-483. doi: https://doi.org/10.1016/j.annepidem.2009.03.021.

\section{ИНФОРМАЦИЯ ОБ АВТОРАХ [AUTHORS INFO]}

*Рожинская Людмила Яковлевна, д.М.н., профессор [Liudmila Y. Rozhinskaya, MD, PhD, Professor]; aдpec: Poсcия, 117036, Москва, ул. Дм. Ульянова, д. 11 [address: 11 Dm.Ulyanova street, 117036 Moscow, Russia]; e-mail: Irozhinskaya@gmail.com; eLibrary SPIN: 5691-7775

Пигарова Екатерина Александровна, д.м.н. [Ekaterina A. Pigarova, MD, PhD]; eLibrary SPIN: 5691-7775; e-mail: k.pigarova@gmail.com

Багрецова Анастасия Александровна [Anastasia A. Bagretsova, MD]

Вербовой Андрей Феликсович, д.м.н., професcop [Andrey F. Verbovoy, MD, PhD, professor]; eLibrary SPIN: $2923-6745$

Кондратьева Наталья Григорьевна [Natalia G. Kondratyeva, MD]

Василевская Ольга Альбертовна [Olga A. Vasilevskaya, MD]

Василюк Василий Богданович, д.М.Н., профессор [Vasily B. Vasilyuk, MD, PhD, professor]; eLibrary SPIN: $1459-5548$

Манько Мария Владимировна, к.м.н. [Maria V. Manko, MD]

Шуньков Виктор Борисович, к.м.н. [Victor B. Shunkov, MD, PhD]

Гребенникова Татьяна Алексеевна, к.M.н. [Tatiana A. Grebennikova, MD, PhD]; ORCID: http://orcid.org/0000-0003-1413-1549; eLibrary SPIN: 4380-5447; e-mail: grebennikova@hotmail.com

\section{ИНФОРМАЦИЯ}

Рукопись получена: 20.12.2020. Одобрена к публикации: 11.01.2021. 


\section{ЦИТИРОВАТЬ:}

Рожинская Л.Я., Пигарова Е.А., Багрецова А.А., Вербовой А.Ф., Кондратьева Н.Г., Василевская О.А., Василюк В.Б., Манько М.В., Шуньков В.Б., Гребенникова Т.А. Применение высокодозных препаратов колекальциферола для лечения дефицита витамина D: результаты открытого многоцентрового сравнительного рандомизированного исследования // Остеопороз и остеonamuu. — 2020. — T. 23. — №3. - C. 4-16. doi: https://doi.org/10.14341/osteo12697

\section{TO CITE THIS ARTICLE:}

Rozhinskaya LY, Pigarova EA, Bagretsova AA, Verbovoy AF, Kondratyeva NG, Vasilevskaya OA, Vasilyuk VB, Manko MV, Shunkov VB, Grebennikova TA. High-doses of cholecalciferol for vitamin D deficiency treatment: results of an open-label, multicenter, comparative, randomized trial. Osteoporosis and bone diseases. 2020;23(3):4-16. doi: https://doi.org/10.14341/osteo12697 